\title{
Electrophysiological Evidences of Inhibition Deficit in Attention-Deficit/ Hyperactivity Disorder During the Attentional Blink
}

\author{
Vladimir López ${ }^{1,2}$, Fabian Pavez ${ }^{1}$, Javier López ${ }^{1}$, Rodrigo Ortega ${ }^{1}$, Nicolas Sáez ${ }^{1}$, \\ Ximena Carrasco ${ }^{1,3}$, Paula Rothhammer ${ }^{1}$ and F. Aboitiz ${ }^{*}, 1$ \\ ${ }^{I}$ Departamento de Psiquiatría y Centro de Investigaciones Médicas, Escuela de Medicina, Pontificia Universidad Cató- \\ lica de Chile, Chile \\ ${ }^{2}$ Escuela de Psicología, Pontificia Universidad Católica de Chile, Chile \\ ${ }^{3}$ Programa de Morfología, Instituto de Ciencias Biomédicas. Facultad de Medicina, Universidad de Chile, Chile
}

\begin{abstract}
Previous studies on the distribution of attentional resources during time have yielded a differential performance in patients with Attention-Deficit/Hyperactivity Disorder (ADHD), in relation to healthy controls. The Attentional Blink (AB) experimental paradigm probes the ability to discriminate visual stimuli in close temporal proximity. The electrophysiological mechanisms underlying the $\mathrm{AB}$ have been intensively studied in healthy individuals, and seems to be very appropriate to evaluate the temporal distribution of attentional resources in ADHD. We recorded Event-Related Potentials (ERPs) to stimuli arranged in a rapid serial visual presentation task. Fourteen male children (age: 11.6 \pm 2.1 ) who met DSM-IV criteria for ADHD of the combined subtype participated in the study, along with fourteen age and sex-matched healthy controls (11.2 \pm 2.3$)$. They were all screened to discard comorbidites. Behavioral responses showed an increased number of commission and omission errors in the ADHD group. Nonetheless, both groups exhibited a significant AB for probes presented in close temporal proximity to targets. ERP waveforms in ADHD showed a P300 component of reduced amplitude, elicited both by detected and undetected probes. However, in the control group the P300 was only elicited by detected probes and its amplitude was larger. As the P3 component is considered to reflect context updating in working memory, its elicitation by missed probes during the attentional blink suggests a failure of an inhibitory selection mechanism in ADHD.
\end{abstract}

\section{INTRODUCTION}

A large body of evidences supports the existence of several cognitive deficits in Attention Deficit Hyperactivity Disorder (ADHD) [1]. This is one of the most common neuropsychiatric diagnoses in childhood, affecting 3-7\% of school-aged children $[2,3]$. Even though the term suggests an attentional abnormality, which indeed becomes evident in some neuropsychological tests, the primary cognitive process affected in the syndrome remains unclear [4]. Most studies of attentional functions on ADHD focus on sustained attention, as measured by Continuous Performing Tests (CPT) [5]. ADHD subjects have been reported to commit an increased number of errors in this type of examination [6].

However, it is well known that paying attention implies more than sustaining attention. Several other subcomponents as selective attention, shifting attention or dividing attention play an important role too [4]. Recent reports on attentional functions in ADHD suggest that some of them are spared in this condition and, even more, that ADHD children can perform better than control subjects in some divided attention tasks $[5,7]$.

\footnotetext{
*Address correspondence to this author at the Departamento de Psiquiatría y Centro de Investigaciones Médicas, Escuela de Medicina, Pontificia Universidad Católica de Chile, Chile; Tel (56-2) 354 3806; Fax: (56-2) 665 1951; E-mail: faboitiz@med.puc.cl
}

Apart from lack of attention, poor performance in CPT and other tests could reflect impairment in other cognitive functions such as vigilance, inhibitory control or working memory [8]. It has been claimed that poor inhibitory control might play a central role in ADHD [2]. This deficiency could explain both the deficits in cognitive functioning and the impulsive behaviors associated with the disorder. Evidence supporting this view has come from neuropsychological data reporting poor performance of ADHD patients in Go- NoGo tasks and the Stroop paradigm [4]. A failure in the inhibition of responses to interfering thoughts or external events could lead to a malfunction of working memory, to a poor goaldirected behavior and to a lack of emotional self-control [4]. While this hypothesis is gaining adepts, the way attentional resources are used and controlled in ADHD, and the participation of an inhibitory deficit in the attentional impairments seen in this condition, are still areas of discussion. In a recent study we have reported a differential pattern of cortical processing in the distribution of visuospatial attentional resources in ADHD [9]. In our spatial task ADHD participants seemed to distribute their attentional resources in a different manner compared to controls, tending to ignore the experimenter's instructions to disregard peripheral stimuli, and following a more scattered pattern of attention. The present study is aiming to explore the same phenomenon, but in the time domain.

The Attentional Blink (AB) refers to a limitation in our ability to attend and correctly identify stimuli presented in rapid succession [10]. It is usually described in a Rapid Se- 
rial Visual Presentation (RSVP) paradigm where trials are composed by a series of stimuli presented in the same spatial location at rates between 6 and 20 items per second [11]. When subjects are asked to identify two targets (T1 and T2) embedded in the stream of stimuli, the accurate identification of the second target T2 (or probe) is impaired if it is presented in close temporal proximity $(200-400 \mathrm{~ms})$ to the (previous) T1 [10-12]. This paradigm seems to be very appropriate to evaluate two crucial aspects in ADHD physiopathology: the distribution of attentional resources in the time domain, and the involvement of working memory and inhibition in ADHD (see below). Previous studies on these patients have found more prolonged $\mathrm{AB}$ periods suggesting a difficulty in voluntarily allocating attention to multiple stimuli separated in time $[8,13,14]$.

Several cognitive models have been proposed to account for the AB. The two-stage model proposes that after a first step of stimulus identification there is a second, limited capacity stage in which the stimulus trace is consolidated in working memory [15]. This second stage would be time consuming and attention demanding. If $\mathrm{T} 2$ is presented while $\mathrm{T} 1$ is still maintained in the limited capacity buffer, its trace is not consolidated and is vulnerable to be overwritten by subsequent stimuli. Another proposed explanation has been termed the interference model [16]. It postulates that missed probes during $\mathrm{AB}$ do reach working memory but are lost due to interferences with T1 [17]. Both models assume a central role of working memory and inhibition processes in the neurophysiological explanation of $\mathrm{AB}$.

Due to its high temporal resolution, the technique of Event Related Potentials (ERPs) has been extensively used to study attention [18]. More specifically, in the Attentional Blink, the P300 component has been one of the most studied. Both $\mathrm{P} 300$ and $\mathrm{AB}$ have been related with frontal function, working memory and inhibitory processes [19-21]. The possibility that the P300 reflects context updating in working memory [19] makes this component an ideal candidate to study the $\mathrm{AB}$ phenomenon. Following this rationale Vogel et al. [17] reported in 1998 that in a RSVP paradigm only those $\mathrm{T} 2$ presented outside the time range of $\mathrm{AB}$ evoked $\mathrm{P} 300$. They interpreted the absence of $\mathrm{P} 300$ during the $\mathrm{AB}$ as evidence that missed $\mathrm{T} 2$ did not reach working memory, favoring the two-stage hypothesis. A subsequent study [22] showed that the previous statement is not true for all probes presented during the AB. Those T2 identified and correctly reported elicited a $\mathrm{P} 300$, while the missed probes failed to evoke the positive component. These results tighten the link between P300, stimulus trace consolidation in working memory, and conscious report of stimulus presence. Recent neuroimaging results [23] also support this view showing that frontal cortex activation is necessary for conscious report of probes presented during $\mathrm{AB}$.

In this study, we took advantage of the ERP technique during the attentional blink paradigm to explore the time domain distribution of attentional resources in ADHD patients. We also assessed the possible implications of inhibition deficit and working memory functions in the emergence of the differential pattern of attention observed in these individuals. If the P300 reflects working memory context updating and cortical disfacilitation to process other stimuli [1921], a different ERP pattern is to be expected in ADHD pa- tients when compared to paired controls during the $\mathrm{AB}$. In normal subjects, the two stage model predicts that missed probes should not elicit a P300 as the stimuli do not access working memory, but according to the interference model they might elicit one because missed $\mathrm{T} 2$ do reach working memory. Likewise, if ADHD implies an inhibitory deficit, then missed probes during the $\mathrm{AB}$ might be able to reach working memory, eliciting a P300 due to context updating.

\section{METHODS}

\section{Participants}

Two groups of subjects were selected to participate in the case-control study:

ADHD group: Fourteen male children who met DSM-IV criteria for ADHD of the combined subtype and had no comorbidity [24]. Their age was $11.6 \pm 2.1$ years $(\mathrm{Mean} \pm \mathrm{SD})$. They were randomly selected out of a larger sample participating in a genetic association study on ADHD. Patients were recruited from general (secondary care) psychiatric and neurological outpatient services. They were asked to interrupt stimulant treatment the day before and the day that the exam was taken. Both, they and their parents agreed to participate in the study and signed a written consent.

Control group: Fourteen age and sex-matched healthy controls. Age: 11.2 \pm 2.3 years. They were selected out of a large group who volunteered for the study from city public schools. No economical reward was given. They underwent a complete physical and psychological examination and were screened using the same instruments as the ADHD group (Conners' test, DSM-IV). They and their parents agreed to participate in the study and signed a written consent.

All participants were Chilean, native Spanish speakers, right handed and had no parental antecedents of left handedness. They had an average or higher IQ, and accepted to be examined to discard any morbidity. The IQ (WISC-R) was not significantly different between the groups: Controls: 110.5 (14.99), ADHD: 112.3 (13.0), $p=0.76$. The experimental procedures were approved by the Ethical Committee of the Faculty of Medicine, Universidad de Chile.

\section{Stimuli and Procedures}

Visual stimulation was accomplished using the STIM system (NeuroScan-Compumedics) synchronized with a digital electroencephalograph. Stimuli consisted of a stream of 12 capital letters presented in a Rapid Serial Visual Presentation (RSVP) as described by Raymond et al. [10]. Visual stimuli were presented on a 21 ' computer monitor placed at a distance of $60 \mathrm{~cm}$ in front of the subject. Monitor refresh rate was $150 \mathrm{~Hz}$. Non target letters were drawn in white over a black background; they were presented at the center of the screen and subtended a visual angle of 3 central degrees. They were all consonants. Target 1 (T1) was drawn in dark red. Target 2 (T2) or Probe was the capital letter X, drawn in white like the rest of the letters.

Each trial was initiated by the subject pressing a key. It began with a fixation cross presented for $200 \mathrm{~ms}$ (Fig. 1). After that, a stream of twelve letters was presented in rapid succession. Each letter was presented for $60 \mathrm{~ms}$ followed by a blank $40 \mathrm{~ms}$ inter-stimulus interval. No letter was repeated 
within a trial. Each trial lasted $1400 \mathrm{~ms}$ not considering the response time window.

In the present study subjects faced three trial conditions: Single Target Task, Dual Target Task and No target Task. These were randomly presented, and although subjects were not informed about the existence of these conditions, they undertook a short training session in which all possible conditions were presented twice. At the end of each trial subjects were asked whether they had identified $\mathrm{T} 1$ and $\mathrm{T} 2$.

The No Target Task consisted of 60 trials in which no Target was presented. The Single Target Task consisted of 60 trials in which only one Target was presented, either T1 or T2 (30 times each). The Dual Target task consisted of 150 trials in which both $\mathrm{T} 1$ and $\mathrm{T} 2$ were present. It was subdivided in 5 conditions according to the relative position of $\mathrm{T} 2$ regarding T1. In this task T1 could appear from position 3 to position 5 in the twelve letters stream. Relative to T1, T2 could appear at five possible positions $(\mathrm{T} 1+2$ to $\mathrm{T} 1+6)$, also referred as lag 2 to lag 6, respectively (Fig. 1). T2 was never the final letter of the stream. Each of these conditions was repeated 30 times, which gave them a relative frequency of occurrence of $25 \%$ regarding no Target and single Target tasks.

Trials from all tasks and conditions were randomly presented throughout the test to counterbalance any list order effect. The experiment was composed by a total of 270 trials divided in 5 blocks of 54 trials each. Between blocks, subjects were allowed to rest or move at their will.

\section{Behavioral Responses}

Subjects' responses at the end of each trial were collected trough the response box of the STIM NeuroScan system. They were saved in a single text file in the stimulation computer. Offline analysis consisted of calculation of Total Number of Errors, Commission Errors defined as responses to non existing Targets in the No Target Task or in the Single Task (reports of T2 when only T1 was present and vice versa) and Omission Errors (No response to a target or no response given in the proper time window).

In the Dual Target task the number of correct identifications of T2 was calculated for each lag or Target relative position. This allowed the estimation of individual performance curves and the identification of the Attentional Blink phenomenon.

\section{Electrophysiological Recordings}

Electrophysiological signals were recorded using a NeuroScan 80-channel Digital Electroencephalograph with high resolution NuAmps amplifiers. An 80 channel cap (QuickCap) from the same company was used for electrode placement.

Impedances were kept below $5 \mathrm{k} \Omega$ throughout the recordings. A/D sampling frequency was set at $250 \mathrm{~Hz}$. A band pass digital filter between 0.1 and $30 \mathrm{~Hz}$ was later applied to remove unwanted frequency components. All EEG channels were recorded referenced to vertex as it is the standard in the Neuroscan System. They were later re-referenced offline to linked mastoids for all further analysis. Two additional bipolar derivations were used to monitor 5 vertical and horizontal ocular movements (EOG). Continuous EEG data were segmented from $200 \mathrm{~ms}$ prior to stimulus to $800 \mathrm{~ms}$ after it. All trials were visually examined to control for artifacts. As usually happens in self-administered tests, there were only a few trials with contamination from eye movements or muscle contraction. All segments with eye movement contamination, or any other technical or biological artifact, were removed from any further analysis. In general rejected trials were less than $5 \%$ in every condition, except in three subjects (2 ADHD, 1 Control) that were excluded from the study due to excessive artifacts. Their data is not included in the sample presented here. A factorial ANOVA considering Group and Condition was used to compare the number of trials remaining after artifact rejection. No significant difference was found between the groups $(F(1,22)=1.07, p>0.23)$. Artifact free segments were averaged to obtain the ERPs. The EEGLAB Matlab toolbox was used for EEG off-line processing and analysis [25].

One ERP waveform was obtained for every condition in each subject. The 'lag 3' condition of the dual task has been reported to represent the time period in which the $\mathrm{AB}$ reaches its maximal intensity $[17,22]$. EEG segments corresponding to this condition were offline classified according to the subject's response into: 'identified T2' and 'missed T2 trials'. They were averaged separately to obtain an individual ERP for these conditions.

Time windows of $50 \mathrm{~ms}$ around the peak latency of the P2 and P300 components were selected to calculate the mean amplitude value in each ERP and condition. These values were used in the statistical analysis. Although the figures show the ERPs group grand averages, all statistical calculations were done using individual waveforms.

\section{Statistical Analysis}

The total number of commission and omission errors was compared between the groups using a $t$ test. A mixed ANOVA design (Group X Lag) with repeated measures in the last factor was used to compare the number of correctly identified T2 at different lags in the Dual Target task.

The individual mean amplitudes of P2 and P300 ERP components were separately compared between groups and conditions using mixed ANOVA designs (Group X Condition X Topography) with repeated measures in the last two factors. Univariate comparisons were done when necessary. Results were corrected with the Greenhouse-Geisser method to adjust the univariate output of repeated measures ANOVA for violations of the compound symmetry assumption.

Six regions of interest (ROI) were defined to represent the scalp topography of the ERP components following a method previously described in AB literature [22]. Groups of electrodes were collapsed into these regions in order to avoid loss of statistical power [26]. These regions were labeled combining the terms: Anterior, Central, Posterior, and Right, Central, Left (AL, AC, AR, CL, CC, CR, PL, PC, and PR). Corresponding electrodes in the 80 channel Neuroscan QuickCap were AL $(1,10,15-17,30-32)$, AC (33-37, 55, 56), AR (52-54, 58-60, 72, 75), CL (2-4, 6-9, 18-20), CC $(28,29,38-40,51,52)$, CR (61-63, 68-71, 76-78), PL (5, 21 26), PC $(27,41-44,49)$ and PR $(46-48,64-67)$. 


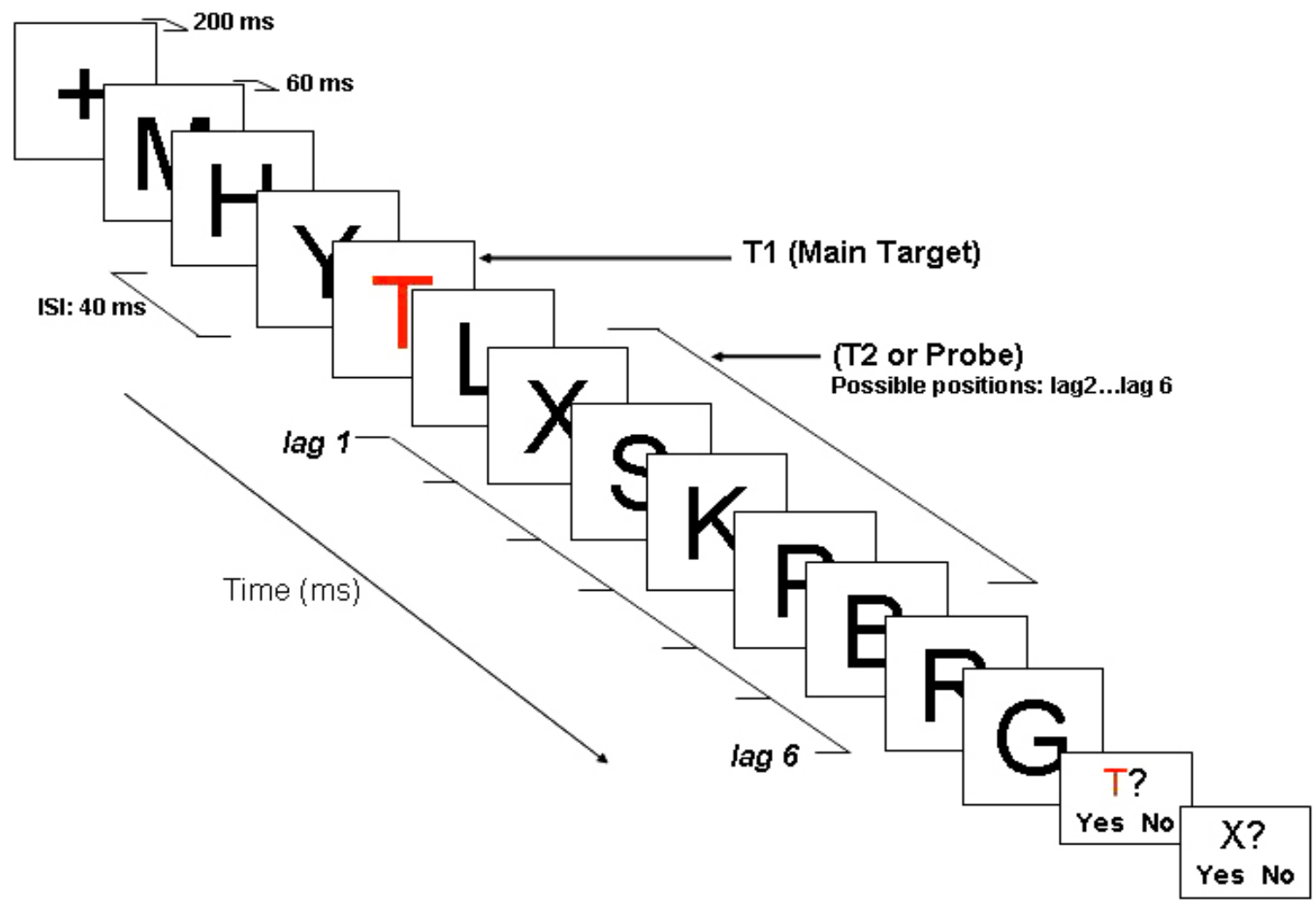

Fig. (1). Dual Task. Each trial consisted of twelve letters presented in rapid succession. The term 'lag' refers to the relative position of the secondary Target (T2) with respect to the main Target (T1). Each lag represents $100 \mathrm{~ms}$ from T1.

\section{RESULTS}

\section{Behavioral Responses}

As expected, the ADHD group evidenced a larger number of both commission $(t=81.4, \mathrm{p}<0.01)$ and omission errors $(t=60.8, \mathrm{p}<0.01)$ compared to controls (Fig. 2). Commission errors were the most frequent in both groups and they were distributed between the No Target task (NT) and the Single Task (ST) as follows: Control $46 \%$ NT and only $54 \%$ ST; ADHD 67\% NT and $43 \%$ ST. In the Dual Target Task the ability to report $\mathrm{T} 2$ depended on its relative position to $\mathrm{T} 1$, showing a significant reduction in lag 2 and lag 3 compared to positions more distant to T1 (Fig. 3). This is the behavioral evidence of the Attentional Blink phenomenon and reaches its maximal intensity in both groups at lag 3 (when T2 is presented approximately $300 \mathrm{~ms}$ after the appearance of T1). The trials included in this analysis were those in which T1 was correctly identified. The repeated measures ANOVA yielded a significant effect for the Factor 'lag' $\left(\mathrm{F}_{(4,130)}=9.87, \mathrm{p}<0.01\right)$. The main factor Group was not significant $\left(\mathrm{F}_{(1,4)}=0.21, \mathrm{p}>0.05\right)$ nor was the group $\mathrm{X}$ lag interaction $\left(\mathrm{F}_{(1,4)}=1.9, \mathrm{p}>0.05\right)$. Paired comparisons showed that the differences in the correct identification of $\mathrm{T} 2$ were mainly between lag 2 and lag 3 on one hand, and the rest of the other lags (4 to 6) on the other $\mathrm{p}<0.05$. The ADHD group had greater variance of responses, especially in lag 4 .

\section{Electrophysiological Data}

Repetitive stimulation of the visual system, beyond $5 \mathrm{~Hz}$, provokes the summation of the early components of Visual Evoked Potentials resulting in a complex waveform named
A Commission Errors

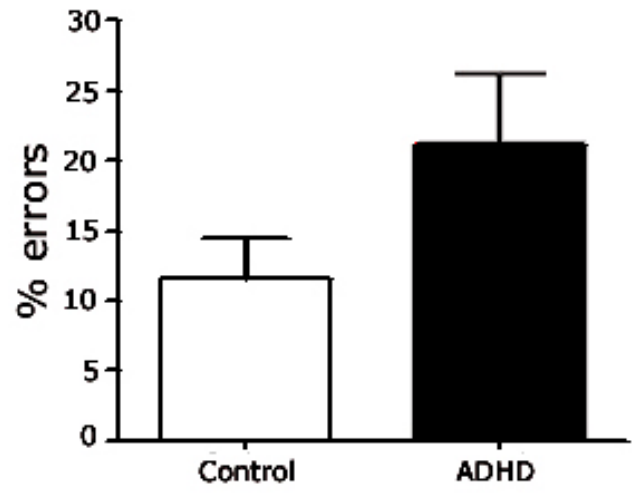

B Omission Errors

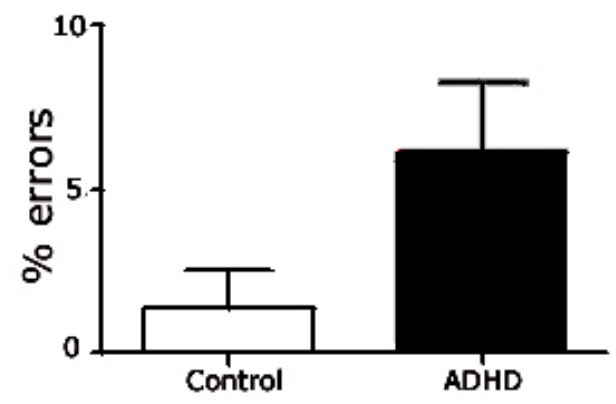

Fig. (2). Percentage of Commission and Omission Errors (Means and Standard Deviations). The percent values refer to the total number of trials in the experiment. 


\section{Probe correct detection. Dual Task}

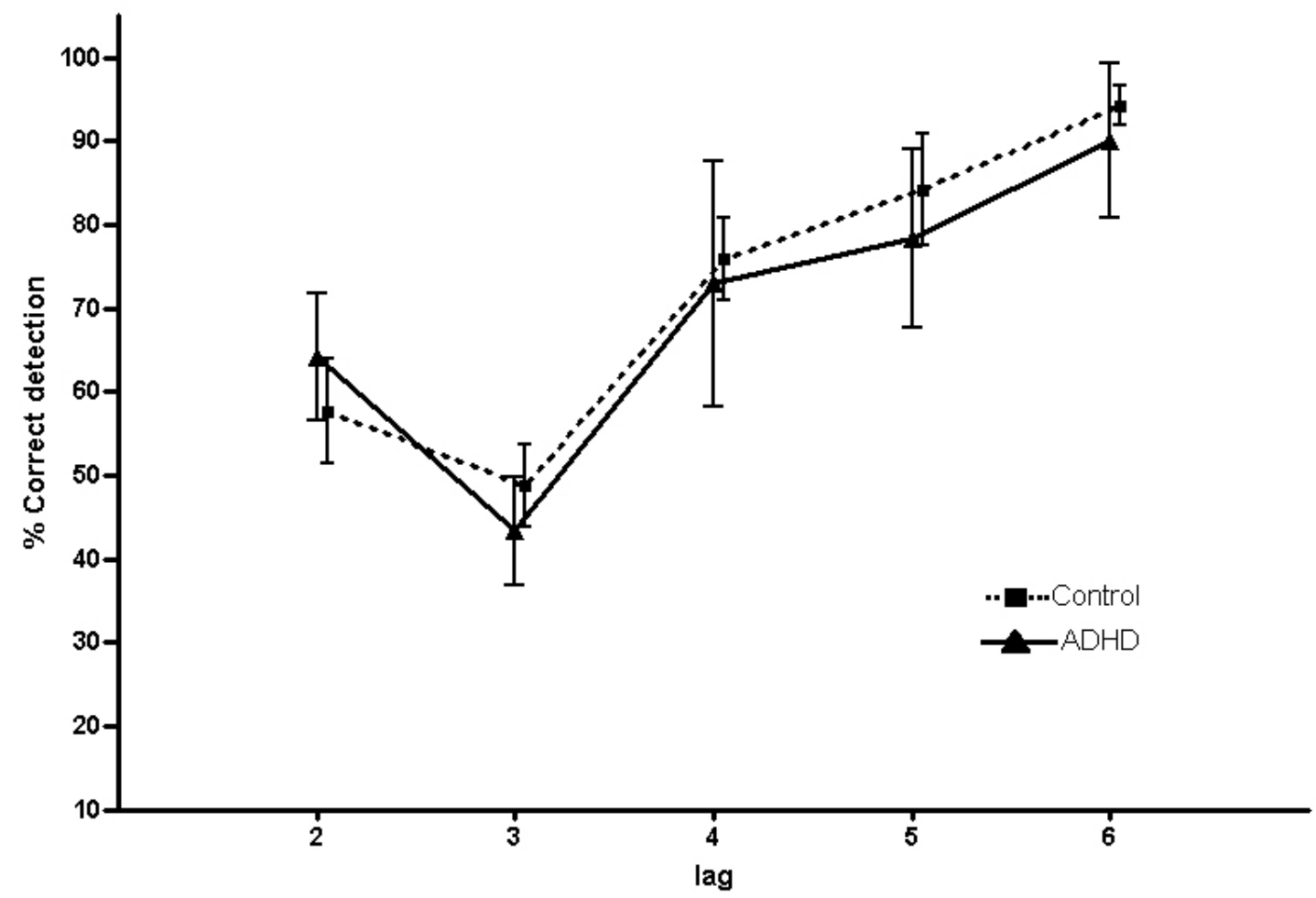

Fig. (3). Percent of correctly identified T2 in the Dual Task condition. Results from lag 2 to lag 6 (200 to 600 ms after T1).

steady state visual evoked potential (ssVEP). This waveform constitutes the baseline for the ERP effects of interest in the present experiment. Any component has to be identified emerging out of this sinusoidal wave.

CONTROL
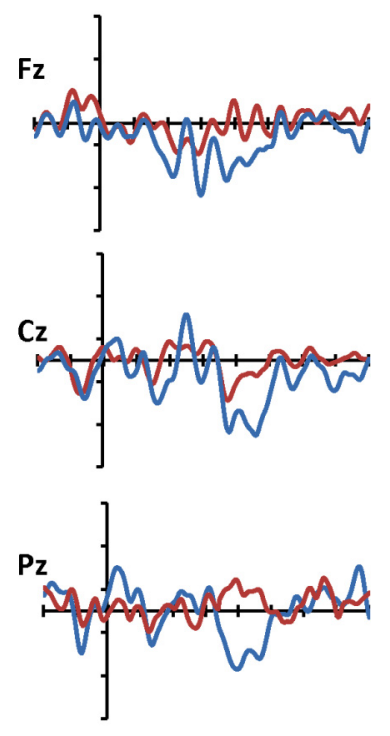

Fig. (4) shows the grand-averages of the responses elicited after T1 occurrence in the single task condition, in each group. A P300 component was clearly seen in both groups with maximal amplitude over the central and parietal regions. This P3 component with a mean latency around 400

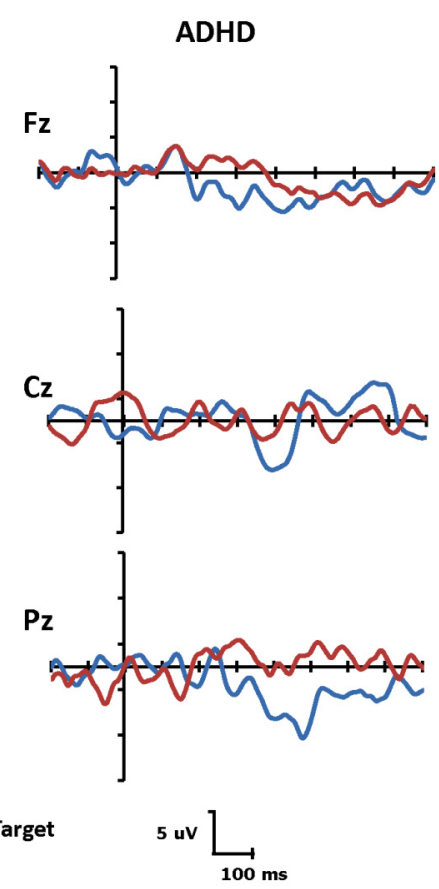

Fig. (4). ERP waveforms elicited by $\mathrm{T} 1$ in the single target task compared to the no target task. Electrode sites Fz, Cz, Pz. Groups ADHD and Control. 

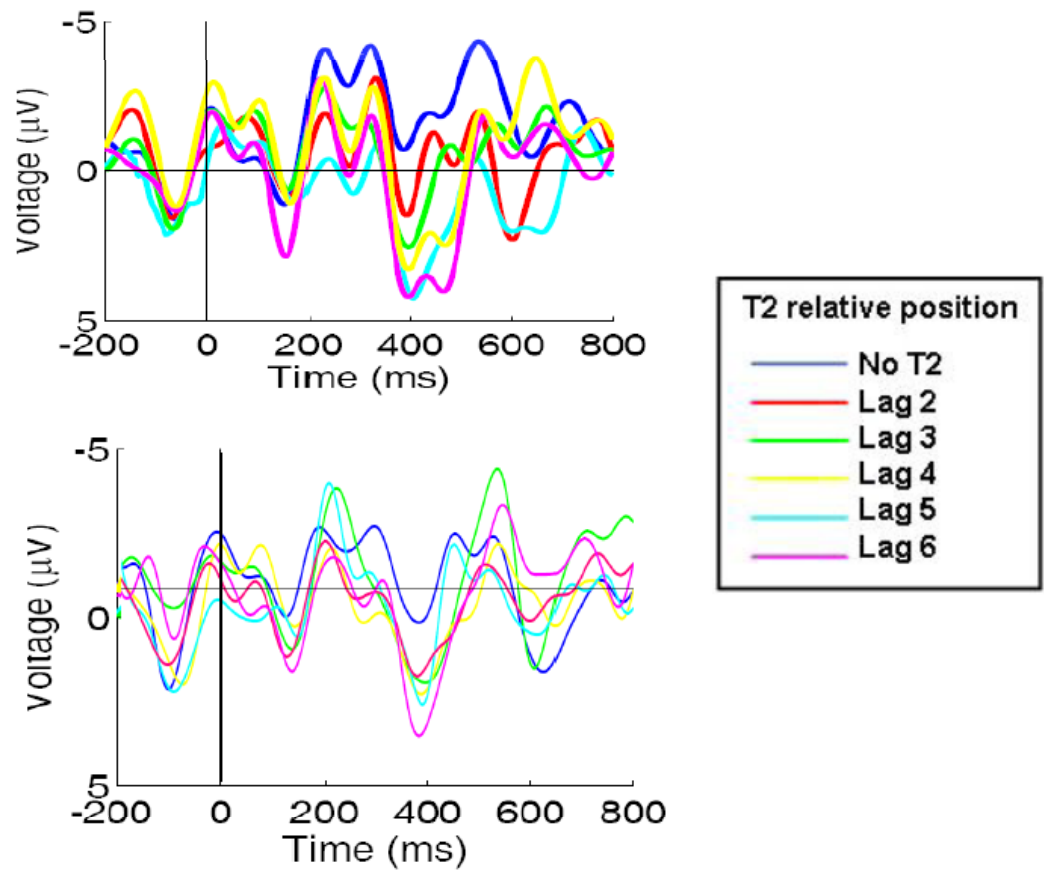

Fig. (5). ERP grand-averages elicited by T2 in the Dual Task (lag 2 to lag 6). Electrode position corresponds to CPz in the 10/10 system. Data include both detected and undetected T2 stimuli. Upper panel, control. Lower panel, ADHD.

ms was significantly larger in the control group compared to ADHD $(F(1,22)=17.64, p<0.01)$. Between-group comparisons of P300 latency and topography were not statistically significant.

The results from the Dual Target Task are, with no doubt, the most important regarding the main questions of this research. Two components are evident in the ERPs elicited by T2 in the Dual Target Task: P200 with an approximate latency of $200 \mathrm{~ms}$ and maximal amplitude over anterior regions, and P300 with peak latency around 400ms and larger amplitudes in central and parietal regions.

ERPs elicited by $\mathrm{T} 2$ in the different conditions of the Dual Target Task (lag 2 to 6) are presented in Fig. (5). In both groups, a positive deflection is evident at around 400 ms after T2. It increases in amplitude from lag 3 to lag 6. The farther T2 is from T1, the larger is the elicited P300. This pattern was more evident in the control group where we found a significant correlation between the variable lag and P300 amplitude over $\mathrm{CPz}$ electrode $(\mathrm{r}=.53, \mathrm{p}<0.05)$. In the ADHD group, the same tendency can be visually identified but it did not reach statistical significance $(\mathrm{r}=.27, \mathrm{p}>0.05)$. The P300 component had larger amplitudes in the Control group compared to ADHD $(\mathrm{F}(1,22)=63.47, \mathrm{p}<0.01)$. No latency shift was statistically significant.

As previously stated, the ERPs elicited by T2 in lag 3 (during maximal AB) were averaged separately according to whether they were identified or not. Figs. (6) and (7) show ERPs to T2 correctly identified and not identified, contrasted with ERPs to the waveform elicited by T2 in the single Target Task, in Controls and ADHD respectively. In both groups a P2 component is evident in ERPs to T2 identified and not identified, but not in the No Target Task condition $\left(\mathrm{F}_{(1,22)}=43.51, \mathrm{p}<0.01\right)$.
The P300 exhibited striking differences between ADHD and Controls. While in the Control group only the T2 correctly identified elicited a large positivity, in the ADHD group both identified and not identified targets elicited significant P300 $\left(\mathrm{F}_{(1,22)}=105.54, \mathrm{p}<0.01\right)$. The interaction Group $\mathrm{X}$ Condition (Identified, not identified) resulted also significant $\left(\mathrm{F}_{(1,22)}=4.549, \mathrm{p}<0.01\right)$.

\section{DISCUSSION}

The distribution of attentional resources in the time domain has been scarcely explored in ADHD. Nevertheless, this approach might shed light on a highly debated issue: the role of inhibitory control in the physiopathology of the syndrome. In the present study we found that T2 reported as not seen during the Attentional Blink do elicit a significant P300 component in ADHD subjects, and not in healthy participants, suggesting a failure to prevent second stimulus information from reaching working memory in this condition.

The behavioral results described here do not differ much of what has been previously reported on ADHD regarding the number of errors in attention demanding tasks [27]. Affected children committed a higher number of errors like in any other Sustained Attention Task $[5,6]$.

The percent of correct identification of a second target, in those trials where T1 was recognized and reported, was significantly reduced when T2 was presented less than $400 \mathrm{~ms}$ after T1, namely the Attentional Blink phenomenon. Previous studies, both in children and adults with the diagnosis of ADHD $[13,14]$, reported a protracted and prolonged AB. No significant differences between the groups were found in the present study regarding $\mathrm{AB}$ duration. However, some of the children from the ADHD group did exhibit a prolonged $\mathrm{AB}$ period. Larger variability in our small sample of ADHD participants may account for the difference between this and previous reports. 


\section{Control Group}
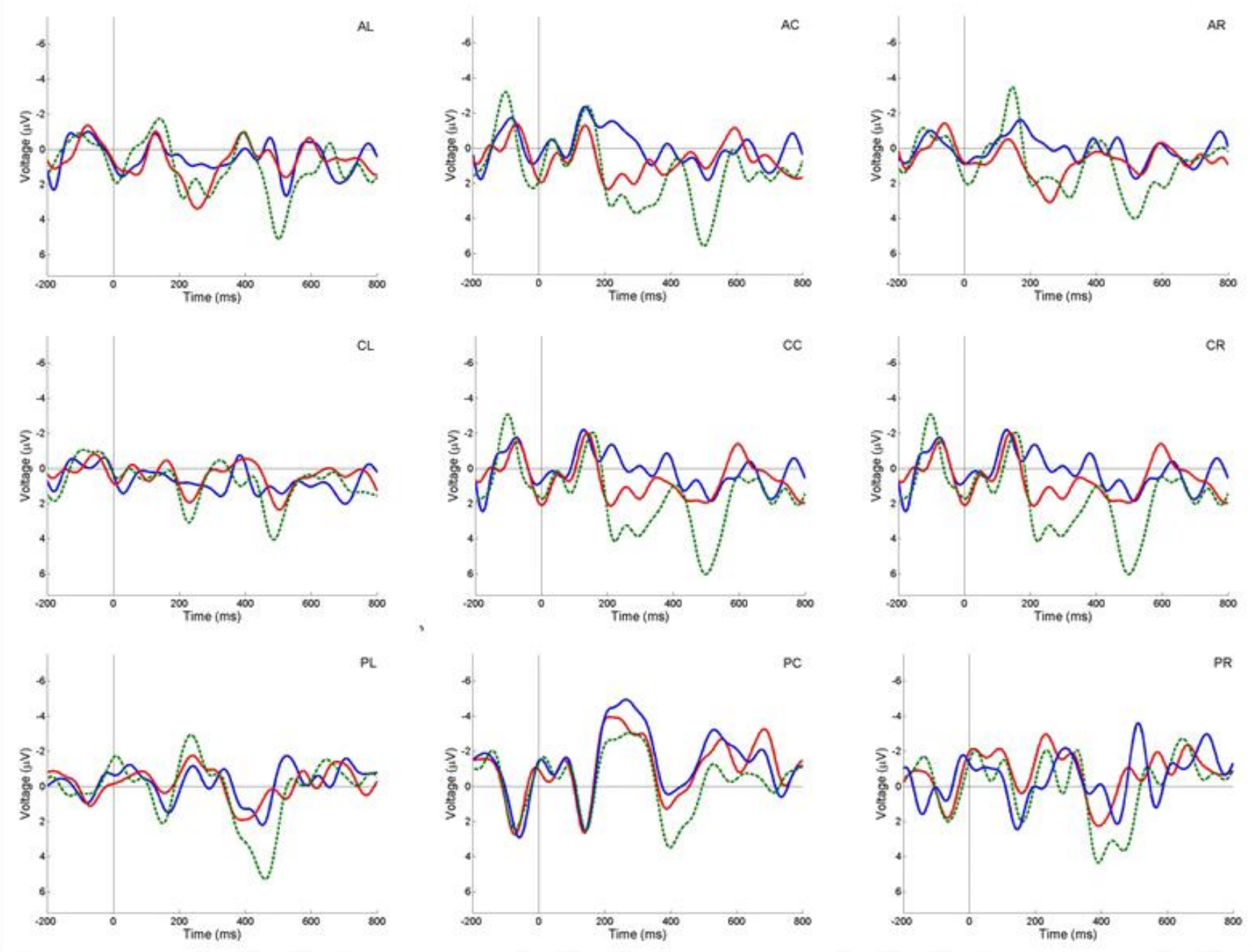

Fig. (6). Control Group. Grand-Average ERPs elicited by T2 in lag 3 of the Dual Task condition contrasted with the Single Task condition (blue). Correctly identified T2 (green - dashed) were averaged separately from missed T2 (red - solid).

A recent study of $\mathrm{AB}$ in children with attention deficithyperactivity disorder [14] suggests that this pattern can be explained in terms of a slower attentional gating. This model assumes that attention acts like a gate controlling the access to the stage of target detection and identification. In ADHD subjects the attention gate would be slower in reopening to allow the processing of the stimuli that follow T1. The stimuli are blocked and blinked out if they appear at a time when the gate is closed.

In this study, ERP waveforms to probes evidenced a socalled P2 or P200, an early positivity with maximal distribution over anterior regions and peaking between 200 and 250 ms. Previous studies [17, 22] have reported a similar finding. The functional significance of this component is not clear yet, although it has been suggested that it reflects perceptual rather than post-perceptual processing [22]. The finding of a P300 of decreased amplitude in the ADHD group has also been widely reported in several ERP experimental paradigms [28]. Interpretations of this reduction generally point to a reduced resource allocation [29]. Interestingly, a reduction of P300 in ADHD subjects was found for the primary task target stimuli of a choice RT task, but not for the subsequent probe (novel) stimuli, suggesting a deficiency in attentional capacity allocation rather than a capacity shortage [30].

Regarding the probes presented during the period of Attentional Blink, Kranczioch et al. [22] demonstrated a nice correlation between identification and correct reports of $\mathrm{T} 2$, and the elicitation of P300. They interpreted this fact as evidence favoring the entry in working memory of information related to these stimuli, which was previously believed not to occur during the $\mathrm{AB}$ [17]. In the present study a very similar pattern of results holds true for healthy individuals from the control group. ADHD participants, nonetheless, exhibited a clearly different pattern. Both identified and not identified T2 elicited significant P300 components.

The possibility that these results could be explained by a random pattern of response regarding $\mathrm{T} 2$ identification can be ruled out considering that $\mathrm{T} 1$ was correctly identified in 


\section{ADHD Group}
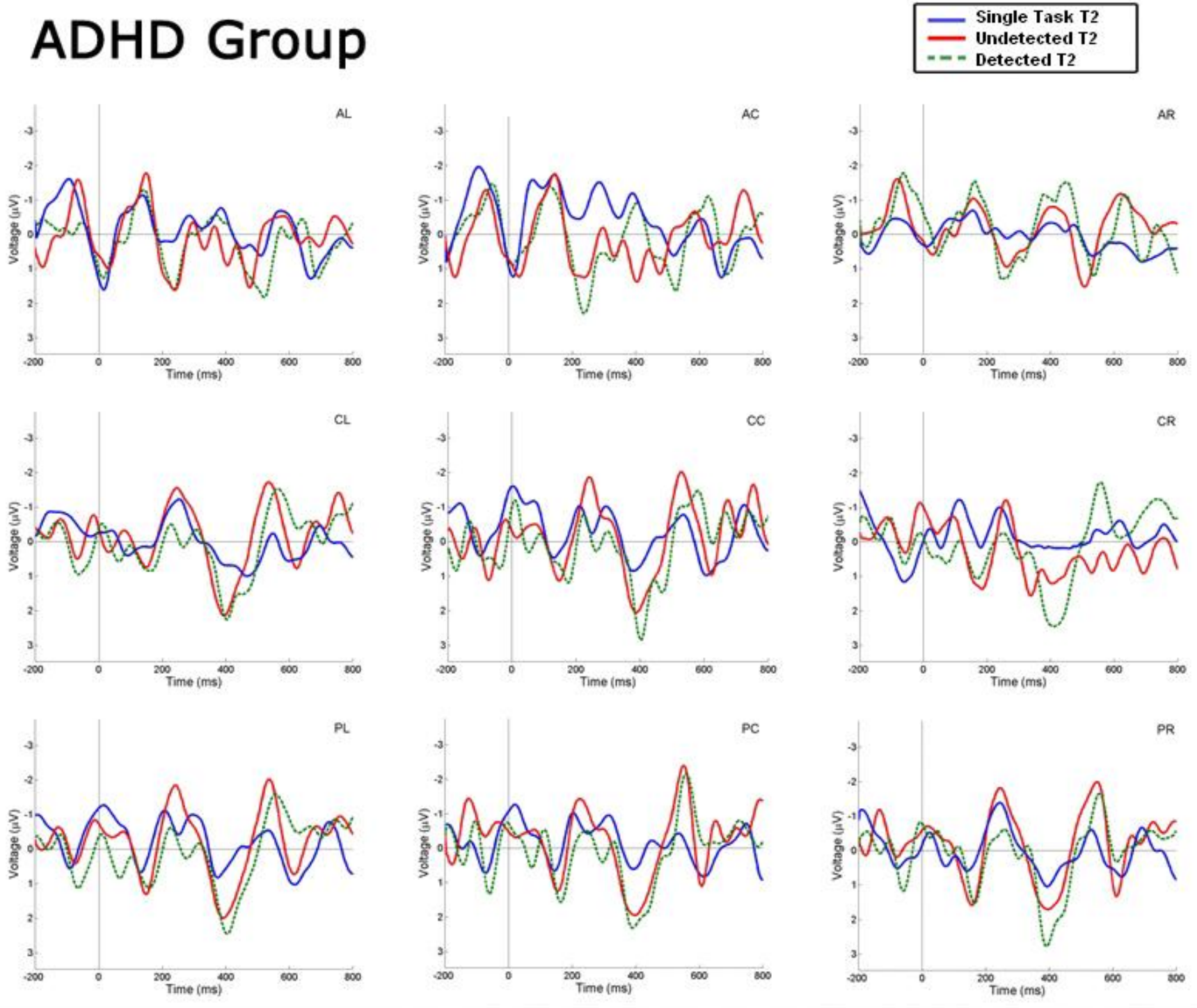

Fig. (7). ADHD Group. Grand-Average ERPs elicited by T2 in lag 3 of the Dual Task condition contrasted with the Single Task condition (blue). Correctly identified T2 (green - dashed) were averaged separately from missed T2 (red - solid).

these trials and that we found no evidence of an arbitrary pattern of response in the rest of the test. Correct identification was, in all cases, above the chance level.

Following the rationale of Kranczioch et al. [22] this finding would suggest that most targets presented during the $\mathrm{AB}$ do reach working memory in ADHD subjects and, therefore, caution should be taken when the usual models are used to explain the blink in this condition. While ERP findings in normal subjects seem to support the 'Two-stage model' [17, 22], the so called 'Interference Model' [16] looks more suitable to account for the present findings in ADHD. The failure to inhibit the entry of the second stimulus into working memory could lead to a competition between targets when the inter-target stimulus-onset asynchrony (SOA) is less than about $500 \mathrm{~ms}$. Limited processing resources initially engaged by $\mathrm{T} 1$ and differential weighting of $\mathrm{T} 1$ over $\mathrm{T} 2$ yields a significant reduction of the capacity to identify and report $\mathrm{T} 2$ after correct detection of T1. In other words, in ADHD subjects, a successful recording of $\mathrm{T} 2$ information into working memory (and therefore a P300 elicitation) does not ensure correct identification and conscious report of the probe; this would depend, as suggested by the Interference Model, on the capacity to succeed in the competition with other items during the retrieval from the short term buffer [16]. This could explain why, in ADHD subjects, probes not reported during the $\mathrm{AB}$ also elicited a $\mathrm{P} 300$. The reduced amplitude of this component in the ADHD group could be reflecting a more distributed pattern of resource allocation, related to a generalized disinhibition of cortical dynamics, which leads to multiple but low-amplitude peaks of activity [31]. Thus, even if the stimulus reaches an active memory buffer, it needs to be also sufficiently robust to elicit conscious report.

In the light of these results, the 'attention gate hypothesis' to account for a prolonged $\mathrm{AB}$ in $\mathrm{ADHD}$, proposed by $\mathrm{Li}$ et al. [14] can be explained in an alternative way. In these patients, the gate could not only be slower in reopening but weaker in general, allowing most items to access and compete for limited processing resources in the detection and 
identification stage. However, in these conditions, entry to the active memory gate may not be sufficient to trigger conscious report, as the stimulus needs to have an adequate salience in relation to competing stimuli. This would represent a different scenario in which the $\mathrm{AB}$ phenomenon could have a different origin and therefore a different intensity and time course.

Aside from these theoretical implications, the present results suggest that ADHD implies a weakened capacity to inhibit stimulus information from reaching working memory. This might explain the poorer performance observed in these patients when a rapid and accurate identification of the stimulus is needed to give the appropriate response or when they have to benefit a stimulus characteristic over another, like in the Stroop Test and Go-NoGo tests [4]. In a parallel work (Lopez et al. 2006) we have found that ADHD children seem to have a different strategy to distribute attentional resources in a visual spatial attention task. In the same line, these results point to a different pattern of control of cognitive resources in this condition. A growing need for a comprehensive understanding of ADHD physiopathology is becoming evident. New or complementary strategies to face this condition should be considered both in educational and health systems.

\section{REFERENCES}

[1] Sergeant J, Geurts H, Oosterlaan J. How specific is a deficit of executive functioning for attention-deficit/hyperactivity disorder? Behav Brain Res 2002; 130: 3-28.

[2] Barkley RA. Behavioral inhibition, sustained attention, and executive functions: Constructing a unifying theory of ADHD. Psychol Bull 1997; 121: 65-94.

[3] Aboitiz F, López V, López-Calderón, J, Carrasco X. Beyond endophenotypes: an interdisciplinary approach to attentional-deficit hyperactivity disorder. In: Vanchevsky MA, Ed. Focus in Cognitive Psychology Research. Chicago: Nova Editorial 2006; pp. 183-205.

[4] Durston S. A review of the biological bases of ADHD: what have we learned from imaging studies? Ment Retard Dev Disabil Res Rev 2003; 9: 184-195.

[5] Koschack J, Kunert HJ, Derichs G, Weniger G, Irle E. Impaired and enhanced attentional function in children with attention deficit / hyperactivity disorder. Psychol Med 2003; 33: 481-489.

[6] Corkum PV, Siegel LS. Is the continuous performance task a valuable research tool for use with children with attention deficit hyperactivity disorder? J Child Psychol Psychiatry 1993; 34: 12171239.

[7] Huang-Pollock CL, Nigg JT. Searching for the attention deficit in attention deficit hyperactivity disorder: The case of visuospatial orienting. Clin Psychol Rev 2003; 23: 801-830.

[8] Hollingsworth DE, McAuliffe SP, Knowlton BJ. Temporal allocation of visual attention in adult attention deficit hyperactivity disorder. J Cogn Neurosci 2001; 13(3): 298-305.

[9] Lopez V, Lopez-Calderon J, Ortega R, Kreither J, Carrasco X, Rothhammer P, Rothhammer F, Rosas R, Aboitiz F. Attentiondeficit hyperactivity disorder involves differential cortical processing in a visual spatial attention paradigm. Clin Neurophysiol 2006; 117(11): 2540-2548.
[10] Raymond JE, Shapiro KL, Arnell KM. Temporary suppression of visual processing in an RSVP task: an attentional blink? J Exp Psychol Hum Percept Perform 1992; 18(3): 849-860.

[11] Sheppard DM, Duncan J, Shapiro KL, Hillstrom AP. Objects and events in the attentional blink. Psychol Sci 2002; 13(5): 410-415.

[12] Vogel EK, Luck SJ. Delayed working memory consolidation during the attentional blink. Psychon Bull Rev 2002; 9(4): 739-743.

[13] Armstrong IT, Munoz DP. Attentional blink in adults with attention-deficit hyperactivity disorder: Influence of eye movements. Exp Brain Res 2003; 152: 243-250.

[14] Li CR, Lin W, Chang H, Hung Y. A psychophysical measure of attention deficit in children with Attention-Deficit/Hyperactivity Disorder. J Abnorm Psychol 2004; 113(2): 228-236.

[15] Chun, MM, Potter MC. A two-stage model for multiple target detection in rapid serial visual presentation. J Exp Psychol Hum Percept Perform 1995; 21(1): 109-127.

[16] Isaac MI, Shapiro KL, Martin J. The attentional blink reflects retrieval competition among multiple rapid serial visual presentation items: tests of an interference model. J Exp Psychol Hum Percept Perform 1999; 25(6): 1774-1792.

[17] Vogel EK, Luck SJ, Shapiro KL. Electrophysiological evidence for a postperceptual locus of suppression during the attentional blink. J Exp Psychol Hum 1998; 24: 1656-1674.

[18] Luck SJ, Woodman GF, Vogel EK. Event-related potential studies of attention. Trends Cogn Sci 2000; 4(11): 432-440.

[19] Donchin E, Coles MGH. Is the P300 component a manifestation of context updating? Behav Brain Sci 1988; 11: 357-427.

[20] Schupp HT, Lutzenberger W, Rau H, Birbaumer N. Positive shifts of event-related potentials: a state of cortical disfacilitation as reflected by the startle reflex probe. Electroencephalogr. Clin Neurophysiol 1994; 90(2): 135-144.

[21] McArthur G, Budd T, Michie P. The Attentional Blink and P300. NeuroReport 1999; 10: 3691-3695.

[22] Kranczioch C, Debener S, Engel, AK. Event-related potential correlates of the attentional blink phenomenon. Cogn Brain Res 2003; 17: $177-187$

[23] Marois R, Yi DJ, Chun MM. The neural fate of consciously perceived and missed events in the attentional blink. Neuron 2004; 41(3): 465-72.

[24] American Psychiatric Association. Diagnostic and Statistical Manual of Mental Disorders, 4th ed. Washington, DC: American Psychiatric Press; 1994.

[25] Delorme A, Makeig S. EEGLAB: an open source toolbox for analysis of single-trial EEG dynamics including independent component analysis. J Neurosci Methods 2004; 134(1): 9-21.

[26] Oken BS, Chiappa KH. Statistical issues concerning computerized analysis of brainwave topography. Ann Neurol 1986; 19: 493-494.

[27] Epstein JN, Conners CK, Erhardt D, March JS, Swanson JM. Asymmetrical Hemispheric control of visual-spatial attention in adults with attention deficit hyperactivity disorder. Neuropsychology 1997; 11: 467-473.

[28] Barry RJ, Johnstone SJ, Clarke AR. A review of electrophysiology in attention-deficit/hyperactivity disorder II. Event-related potentials. Clin Neurophysiol 2003; 114: 184-198.

[29] Steger J, Imhof K, Steinhausen HC, Brandeis D. Brain mapping of bilateral interactions in attention deficit hyperactivity disorder and control boys. Clin Neurophysiol 2000; 111: 1141-1156.

[30] Jonkman LM, Kemner C, Verbaten MN, et al. Attentional capacity, a probe ERP study: differences between children with attentiondeficit hyperactivity disorder and normal control children and effects of methylphenidate. Psychophysiology 2000; 37: 334-346.

[31] Seamans JK, Yang CR. The principal features and mechanisms of dopamine modulation in the prefrontal cortex. Prog Neurobiol 2004; 74(1): $1-58$ 